\title{
On the approximation exponent of some hyperquadratic power series
}

\author{
Khalil Ayadi
}

\begin{abstract}
In this paper, we give the value of the approximation exponent of the hyperquadratic power series satisfying the equation

$$
C x^{r}-A x^{r-1}-1=0
$$

where $r>2$ is a power of a prime number $p, A$ and $C$ are nonzero polynomials over a finite field $\mathbf{K}$ of characteristic $p$ and $\operatorname{deg} A>\operatorname{deg} C$. Further, we exhibit explicitly its continued fraction expansion when $C$ divides $A$.
\end{abstract}

\section{Introduction}

Let $p$ be a given prime number and $\mathbf{K}$ be a finite field of characteristic $p$. We denote by $\mathbf{K}[X]$ the ring of polynomials with coefficients in $\mathbf{K}$ and $\mathbf{K}(X)$ the field of fractions of $\mathbf{K}[X]$. Let $\mathbf{K}\left(\left(X^{-1}\right)\right)$ be the field of formal power series:

$$
\mathbf{K}\left(\left(X^{-1}\right)\right)=\left\{\alpha=\sum_{i \leq n_{0}} u_{i} X^{i} \quad: \quad n_{0} \in \mathbb{Z} \quad \text { and } \quad u_{i} \in \mathbf{K}\right\}
$$

Let $\alpha=\sum u_{i} X^{i}$ be any formal power series, we define its polynomial part, denoted $[\alpha]$, by $[\alpha]:=\sum_{i \geq 0} u_{i} X^{i}$. If $\alpha \neq 0$, then the degree of $\alpha$ is $\operatorname{deg}(\alpha)=$ $\sup \left\{i: u_{i} \neq 0\right\}$ and $\operatorname{deg}(0)=-\infty$. Thus, we define the not archimedean absolute value over $\mathbf{K}\left(\left(X^{-1}\right)\right)$ by $|\alpha|=|X|^{\operatorname{deg}(\alpha)}$ where $|X|>1$, and $|0|=0$.

Received by the editors in October 2014.

Communicated by A. Weiermann.

2010 Mathematics Subject Classification : 11J61,11J70.

Key words and phrases : diophantine approximation, formal power series, continued fraction. 
There is a strong analogy between the classical construction of the field of real numbers and the field of power series which we are considering here. The role of $\{ \pm 1\}, \mathbb{Z}, \mathbb{Q}$ and $\mathbb{R}$ are played by $\mathbf{K}^{*}, \mathbf{K}[X], \mathbf{K}(X)$ and $\mathbf{K}\left(\left(X^{-1}\right)\right)$. As in the classical context of real numbers, we have a continued fraction algorithm in $\mathbf{K}\left(\left(X^{-1}\right)\right)$. If $\alpha \in \mathbf{K}\left(\left(X^{-1}\right)\right)$ we can write

$$
\alpha=a_{0}+\frac{1}{a_{1}+\frac{1}{a_{2}+\frac{1}{\ddots}}}=\left[a_{0}, a_{1}, a_{2}, \ldots\right]
$$

where $a_{i} \in \mathbf{K}[X]$. The $a_{i}$ are called the partial quotients and we have $\operatorname{deg} a_{i}>0$ for $i>0$. This continued fraction is finite if and only if $\alpha \in \mathbf{K}(X)$. We define two sequences of polynomial $\left(P_{n}\right)$ and $\left(Q_{n}\right)$ by $P_{0}=a_{0}, Q_{0}=1, P_{1}=a_{0} a_{1}+1$, $Q_{1}=a_{1}$ and for any $n \geq 2$,

$$
P_{n}=a_{n} P_{n-1}+P_{n-2}, \quad Q_{n}=a_{n} Q_{n-1}+Q_{n-2} .
$$

$\frac{P_{n}}{Q_{n}}=\left[a_{0}, a_{1}, a_{2} \ldots, a_{n}\right]$ is called the $n^{\text {th }}$-convergent of $\alpha$ and we have $P_{n} Q_{n-1}-P_{n-1} Q_{n}=(-1)^{n-1}$. Further, we have the following important equality

$$
\left|\alpha-\frac{P_{n}}{Q_{n}}\right|=\left|a_{n+1}\right|^{-1}\left|Q_{n}\right|^{-2} . \quad(*)
$$

One of the basic question in Diophantine approximation is how the irrational elements of $\mathbf{K}\left(\left(X^{-1}\right)\right)$ can be approximated by rational elements. Our aim is to study the irrationality exponent of power series that are algebraic over the field of rational functions. In order to measure the quality of rational approximation, we introduce the following notation and definition. Let $\alpha$ be an irrational element of $\mathbf{K}\left(\left(X^{-1}\right)\right)$.

For all real numbers $\mu$, we define

$$
B(\alpha, \mu)=\liminf _{|Q| \rightarrow \infty}|Q|^{\mu}|Q \alpha-P|
$$

where $P$ and $Q$ run over polynomials in $\mathbf{K}[X]$ with $Q \neq 0$. Now the approximation exponent of $\alpha$ is defined by

$$
v(\alpha)=\sup \{\mu \in \mathbb{R}: B(\alpha, \mu)<\infty\}
$$

Note that if $\frac{P_{n}}{Q_{n}}$ is a convergent to $\alpha$ then the equality $(*)$ gives that

$$
\left|Q_{n} \alpha-P_{n}\right|=\left|Q_{n}\right|^{-\left(\frac{\operatorname{deg} Q_{n+1}}{\operatorname{deg} Q_{n}}\right)} .
$$

Since the best rational approximation to $\alpha$ are its convergents, with the above notation, we have

$$
v(\alpha)=\limsup \left(\frac{\operatorname{deg} Q_{k+1}}{\operatorname{deg} Q_{k}}\right)=1+\lim \sup \left(\frac{\operatorname{deg} a_{k+1}}{\sum_{1 \leq i \leq k} \operatorname{deg} a_{i}}\right) .
$$


It is clear that the approximation exponent can be determined when the continued fraction of the element is explicitly known. Since $\left|Q_{n} \alpha-P_{n}\right| \leq\left|Q_{n}\right|^{-1}$, for all irrational $\alpha \in \mathbf{K}\left(\left(X^{-1}\right)\right)$ we have $v(\alpha) \geq 1$. Furthermore Mahler's version of Liouville's Theorem says that if $\alpha \in \mathbf{K}\left(\left(X^{-1}\right)\right)$ is algebraic over $\mathbf{K}(X)$ of degree $n>1$ then $B(\alpha, n-1) \neq 0$. Consequently, for $\alpha \in \mathbf{K}\left(\left(X^{-1}\right)\right)$ algebraic over $\mathbf{K}(X)$ of degree $n>1$ we have $v(\alpha) \in[1, n-1]$.

We say that $\alpha \in \mathbf{K}\left(\left(X^{-1}\right)\right)$ is badly approximable by rational elements, which is equivalent to saying that $\alpha$ admits bounded partial quotients if $v(\alpha)=1$ and $B(\alpha, 1) \neq 0$. We also say that $\alpha \in \mathbf{K}\left(\left(X^{-1}\right)\right)$ is well approximable by rationales, which is equivalent to saying that $\alpha$ admits unbounded partial quotients if $v(\alpha)>1$. The reader who is interested in a survey on the different contributions to this topic and for full references can consult for example [2], [9] and [[10], Chap. 9].

We define now a specific class of power series noted by $\mathcal{H}$, which is called the class of hyperquadratic. It contains the irrational elements $\alpha$ in $\mathbf{K}\left(\left(X^{-1}\right)\right)$, satisfying an algebraic equation of the form

$$
x=\frac{A x^{r}+B}{C x^{r}+D}
$$

where $A, B, C, D \in \mathbf{K}[X]$ and $r=p^{t}, t \geq 0$. A famous example of well approximable element in $\mathbb{F}_{p}\left(\left(X^{-1}\right)\right)$ is given by K. Mahler [3] in 1949, which belongs to $\mathcal{H}$, and satisfies the algebraic equation $\alpha=X^{-1}+\alpha^{p}$.

It gradually became apparent that the elements of class $\mathcal{H}$ deserve special consideration.

Rational approximation of elements of class $\mathcal{H}$ has been studied also by J. Voloch [11] and more deeply by B. de Mathan [4]. They could show that if the partial quotients in the continued fraction expansion of such elements $\alpha$ are unbounded, then $v(\alpha)>1$. By the work of B.de Mathan [4], we know moreover that for elements of class $\mathcal{H}$, the approximation exponent $v(\alpha)$ is a rational number and $B(\alpha, v(\alpha)) \neq 0, \infty$. The possibility of describing the two subsets of $\mathcal{H}$, formed on the one hand by badly approximable elements and on the other hand by well approximable elements remain open.

Now we will show how it is possible in some cases to compute the approximation exponent for an algebraic element, without knowing the whole continued fraction. This will be possible if this approximation exponent is large enough, that is to say not close to 1. A. Lasjaunias [2] has given applications to algebraic elements which are of class $\mathcal{H}$ and also to others which are not. The basic idea in the following result is due to J. Voloch [11]. We state below an improved version derived from B. de Mathan [5].

Theorem 1.1. Let $\alpha \in \mathbf{K}\left(\left(X^{-1}\right)\right)$. Assume that there is a sequence $\left(P_{n}, Q_{n}\right)_{n \geq 0}$, with $P_{n}, Q_{n} \in \mathbf{K}[X]$, satisfying the following conditions:

(1) There are two real constants $\lambda>0$ et $\mu>1$, such that

$$
\left|Q_{n}\right|=\lambda\left|Q_{n-1}\right|^{\mu} \text { and }\left|Q_{n}\right|>\left|Q_{n-1}\right| \text { for all } n \geq 1 \text {. }
$$


(2) There are two real constants $\rho>0$ and $\gamma>1+\sqrt{\mu}$, such that

$$
\left|\alpha-\frac{P_{n}}{Q_{n}}\right|=\rho\left|Q_{n}\right|^{-\gamma} \text { for all } n \geq 0 \text {. }
$$

Then we have $v(\alpha)=\gamma-1$. Further, if $\operatorname{gcd}\left(P_{n}, Q_{n}\right)=1$ for $n \geq 0$, we have $B(\alpha, v(\alpha))=\rho$, and if the sequence $\left(\operatorname{gcd}\left(P_{n}, Q_{n}\right)\right)_{n \geq 0}$ is bounded then $B(\alpha, v(\alpha)) \neq$ $0, \infty$.

In this work, we consider an irreducible equation of the form

$$
C x^{r}-A x^{r-1}-1=0
$$

where $r>2$ is a power of $p, A$ and $C$ are nonzero polynomial with coefficients in $\mathbf{K}$ such that $\operatorname{deg} A>\operatorname{deg} C$. Note that the case $C \in \mathbf{K}^{*}$ was studied by $\mathbf{W}$. Schmidt (see [9]. p 158). This equation has a unique solution of strictly positive degree, furthermore, if we note by $\alpha$ this solution then $[\alpha]=\left[\frac{A}{C}\right]$ (see [8]. p 243). Note that the equation (1) satisfied by $\alpha$ can be written as $x=A x^{r} /\left(C x^{r}-1\right)$, so $\alpha$ is an hyperquadratic power series and its approximation exponent belongs to $[1, r-1]$.

We are interested on computing the approximation exponent of $\alpha$. Further, we describe its continued fraction expansion when $C$ divides $A$. For this, we recall the following notations. Let $P_{n} / Q_{n} \in \mathbf{K}(X)$ such that $P_{n} / Q_{n}:=\left[a_{1}, a_{2}, \ldots, a_{n}\right]$. For all $x \in \mathbf{K}(X)$, we will note

$$
\left[\left[a_{1}, a_{2}, \ldots, a_{n}\right], x\right]:=\frac{P_{n}}{Q_{n}}+\frac{1}{x}
$$

We state now a basic and technical Lemma concerning continued fractions. The idea involved in this Lemma appears for the first time in works of M. Mendes France [6] on finite continued fraction in the context of real numbers.

Lemma 1.2. Let $a_{1}, \ldots, a_{n}, x \in \mathbb{F}_{q}(X)$. We have the following equality:

$$
\left[\left[a_{1}, a_{2}, \ldots, a_{n}\right], x\right]=\left[a_{1}, a_{2}, \ldots, a_{n}, y\right] \text {, where } y=(-1)^{n-1} Q_{n}^{-2} x-Q_{n-1} Q_{n}^{-1} \text {. }
$$

Particularly we have

$$
\left[\left[a_{1}, a_{2}\right], x\right]=\left[a_{1}, a_{2}, y\right] \text {, where } y=-a_{2}^{-2} x-a_{2}^{-1} .
$$

The proof of this Lemma can be found in Lasjaunias's paper [1].

\section{Results}

Theorem 1.1 allows us to compute the approximation exponent of well approximable formal series. We obtain as application of this Theorem, interesting results for the approximation exponent of the solution of the equation (1) by giving a precise value of the exponent. 
Theorem 2.1. Let $\alpha$ be the irrational solution of equation (1) such that $\operatorname{gcd}(A, C)=1$. Suppose that $|\alpha|=|C|^{s}$ with $s>\frac{\sqrt{r}}{r-\sqrt{r}-1}$. Then

$$
v(\alpha)=\frac{s r+1}{s+1} \text { and } B(\alpha, v(\alpha))=|C|^{-\frac{s^{2}\left(r^{2}-r\right)}{s+1}}
$$

Proof.

We consider the following sequence: $P_{0}=A, Q_{0}=C$ and for $n \geq 1$

$$
\begin{gathered}
P_{n}=A P_{n-1}^{r} \\
Q_{n}=C P_{n-1}^{r}+Q_{n-1}^{r} .
\end{gathered}
$$

Then for all $n \geq 0$ :

$\left|\alpha-\frac{P_{n}}{Q_{n}}\right|=\left|\frac{A \alpha^{r}}{C \alpha^{r}+1}-\frac{A P_{n-1}^{r}}{C P_{n-1}^{r}+Q_{n-1}^{r}}\right|=\frac{|A|\left|\alpha^{r} Q_{n-1}^{r}-P_{n-1}^{r}\right|}{|C||\alpha|^{r}|C|\left|P_{n-1}^{r}\right|}=$ $\frac{1}{|C||\alpha|^{2 r-1}}\left|\alpha-\frac{P_{n-1}}{Q_{n-1}}\right|^{r}$.

We show by recursion that for all $n \geq 0$ :

$$
\left|\alpha-\frac{P_{n}}{Q_{n}}\right|=|C|^{-\frac{\left(r^{n}-1\right)}{r-1}}|\alpha|^{-\frac{(2 r-1)\left(r^{n}-1\right)}{r-1}}\left|\alpha-\frac{P_{0}}{Q_{0}}\right|^{r^{n}}
$$

since $\left|\alpha-\frac{P_{0}}{Q_{0}}\right|=\left|\alpha-\frac{A}{C}\right|=\frac{|A|}{|C||C||\alpha|^{r}}=\frac{1}{|C||\alpha|^{(r-1)}}$ then $\left|\alpha-\frac{P_{0}}{Q_{0}}\right|^{r^{n}}=$ $|C|^{-r^{n}}|\alpha|^{-r^{n}(r-1)}$. So

$$
\left|\alpha-\frac{P_{n}}{Q_{n}}\right|=|C|^{-\frac{r^{n+1}-1}{r-1}}|\alpha|^{-\frac{r^{n+2}-2 r+1}{r-1}}=|C|^{-\frac{s r^{n+2}+r^{n+1}-2 s r+s-1}{r-1}} .
$$

Secondly, we have for all $n \geq 1 Q_{n}=C P_{n-1}^{r}+Q_{n-1}^{r}$ and since $\left|P_{n-1}\right|=|C|^{s}\left|Q_{n-1}\right|$ then

$$
\left|Q_{n}\right|=|C|^{s r+1}\left|Q_{n-1}\right|^{r} .
$$

Again by recursion we show that

$$
\left|Q_{n}\right|=|C|^{\frac{(s r+1)\left(r^{n}-1\right)}{r-1}}\left|Q_{0}\right|^{r^{n}}=|C|^{\frac{(s+1) r^{n+1}-s r-1}{r-1}} .
$$

So we obtain for all $n \geq 0$ :

$$
\left|\alpha-\frac{P_{n}}{Q_{n}}\right|=|C|^{-\frac{s^{2}\left(r^{2}-r\right)}{s+1}}\left|Q_{n}\right|^{-\frac{s r+1}{s+1}} .
$$

We can verifies that if $s>\frac{\sqrt{r}}{r-\sqrt{r}-1}$ then $\frac{s r+1}{s+1}>1+\sqrt{r}$. Hence by Theorem 1.1 we conclude that $v(\alpha)=\frac{s(r-1)}{s+1}$. Further, since $\operatorname{gcd}(A, C)=1$ then $\operatorname{gcd}\left(P_{n}, Q_{n}\right)=1$ for all $n \geq 0$ and so $B(\alpha, v(\alpha))=|C|^{-\frac{s^{2}\left(r^{2}-r\right)}{s+1}}$.

Note that, in this Theorem, the condition $|\alpha|=|C|^{s}$ together with $s>\frac{\sqrt{r}}{r-\sqrt{r}-1}$ are obtained if $(r-\sqrt{r}-1) \operatorname{deg} A>(r-1) \operatorname{deg} C$. 
Example 2.1. Let $\alpha \in \mathbb{F}_{5}\left(\left(X^{-1}\right)\right)$ be the irrational solution of strictly positive degree of the equation:

$$
\left(X^{2}-2\right) \alpha^{5}+X^{5} \alpha^{4}-1=0
$$

We have $|\alpha|=|X|^{3}=\left|X^{2}-2\right|^{\frac{3}{2}}$. Since $\frac{3}{2}>\frac{\sqrt{5}}{5-\sqrt{5}-1}$, then we get that $v(\alpha)=\frac{12}{5}$.

Now, we will see the case when $C$ divides $A$. For this case, we will give explicitly the continued fraction expansion for the solution of the equation (1). Knowing all the partial quotients of the solution of (1), we can compute the exact value of its approximation exponent.

Theorem 2.2. Let $\alpha$ be the irrational solution of the equation (1). Assume that $C$ divides A. Then

$$
\alpha=\left[a_{0}, \cdots, a_{n}, \cdots\right]
$$

where $a_{0}=A / C$ and for all $n \geq 0$ :

$$
\begin{gathered}
a_{2 n}=(-1)^{n} a_{0}, \\
a_{2 n+1}= \begin{cases}-C a_{0}^{-1} a_{n}^{r} & \text { if } n \text { is even; } \\
-a_{n}^{r} / a_{0} C & \text { if } n \text { is odd. }\end{cases}
\end{gathered}
$$

Furthermore, $v(\alpha)=r-1$.

Proof. It is clear that if $C$ divides $A$ then the first partial quotient of $\alpha$ is $a_{0}=A / C$ and $\alpha=a_{0}+\frac{1}{\alpha_{1}}$. If $\alpha$ is a solution of $(1)$ then we have

$$
\alpha^{r}=\frac{\alpha}{-A+C \alpha}=\frac{a_{0} \alpha_{1}+1}{C} .
$$

Then $C \alpha^{r}=a_{0} \alpha_{1}+1$. So $C a_{0}^{r}+\frac{C}{\alpha_{1}^{r}}=a_{0} \alpha_{1}+1$. This implies that

$$
\left[\left[C a_{0}^{r-1},-a_{0}\right], \frac{a_{0} \alpha_{1}^{r}}{C}\right]=\alpha_{1}
$$

then, from Lemma 1.2, we obtain that $\left[C a_{0}^{r-1},-a_{0}, \alpha_{3}\right]=\alpha_{1}$. Hence $a_{1}=C a_{0}^{r-1}$ (since $\operatorname{deg} C a_{0}^{r-1}>0$ ) and $a_{2}=-a_{0}$ and $\alpha_{3}=-\frac{a_{0} \alpha_{1}^{r}}{a_{0}^{2} C}+\frac{1}{a_{0}}$. We apply again the same reasoning and we obtain $\alpha_{3}=\frac{-C^{r} a_{0}^{r^{2}-r}}{a_{0} C}+\frac{1}{a_{0}}-\frac{1}{a_{0} C \alpha_{2}^{r}}$. Hence

$$
\alpha_{3}=\left[\left[-C^{r-1} a_{0}^{r^{2}-r-1}, a_{0}\right],-a_{0} C \alpha_{2}^{r}\right]=\left[-C^{r-1} a_{0}^{r^{2}-r-1}, a_{0}, \alpha_{5}\right]
$$

with

$$
\alpha_{5}=\frac{a_{0} C \alpha_{2}^{r}}{a_{0}^{2}}-\frac{1}{a_{0}}=\frac{C}{a_{0}} \alpha_{2}^{r}-\frac{1}{a_{0}}
$$


We get $a_{3}=-C^{r-1} a_{0}^{r^{2}-r-1}, a_{4}=a_{0}$. Further

$$
\alpha_{5}=\frac{C}{a_{0}} a_{2}^{r}+\frac{C}{a_{0} \alpha_{3}^{r}}-\frac{1}{a_{0}}=-C a_{0}^{r-1}+\frac{C}{a_{0} \alpha_{3}^{r}}-\frac{1}{a_{0}} .
$$

So

$$
\alpha_{5}=\left[\left[-C a_{0}^{r-1},-a_{0}\right], \frac{a_{0} \alpha_{3}^{r}}{C}\right]=\left[-C a_{0}^{r-1},-a_{0}, \alpha_{7}\right],
$$

with $\alpha_{7}=-\frac{a_{0} \alpha_{3}^{r}}{a_{0}^{2} C}+\frac{1}{a_{0}}$. Then $a_{5}=-C a_{0}^{r-1}, a_{6}=-a_{0}$. Further

$$
\begin{aligned}
\alpha_{7}=-\frac{a_{3}^{r}}{a_{0} C}+\frac{1}{a_{0}}-\frac{1}{a_{0} C \alpha_{4}^{r}}=\left[\left[-C^{r^{2}-r-1} a_{0}^{r^{3}-r^{2}-r-1}, a_{0}\right],-a_{0} C \alpha_{4}^{r}\right]= \\
\\
\quad\left[-C^{r^{2}-r-1} a_{0}^{r^{3}-r^{2}-r-1}, a_{0}, \alpha_{9}\right]
\end{aligned}
$$

So $a_{7}=-C^{r^{2}-r-1} a_{0}^{r^{3}-r^{2}-r-1}, a_{8}=a_{0}$ and

$$
\begin{gathered}
\alpha_{9}=-\frac{a_{0} C \alpha_{4}^{r}}{a_{0}^{2}}-\frac{1}{a_{0}}=\frac{C}{a_{0}} \alpha_{4}^{r}-\frac{1}{a_{0}} . \\
=\frac{C}{a_{0}} a_{4}^{r}-\frac{1}{a_{0}}+\frac{C}{a_{0} \alpha_{5}^{r}} .
\end{gathered}
$$

Then again

$$
\alpha_{9}=\left[\left[C a_{0}^{r-1},-a_{0}\right], \frac{a_{0} \alpha_{5}^{r}}{C}\right]=\left[C a_{0}^{r-1},-a_{0}, \alpha_{11}\right]
$$

with $a_{9}=C a_{0}^{r-1}, a_{10}=-a_{0}$ and $\alpha_{11}=\frac{-\alpha_{5}^{r}}{a_{0} C}+\frac{1}{a_{0}}$. This gives that $a_{11}=-C^{r-1} a_{0}^{r^{2}-r-1}, a_{12}=a_{0}$, so one.

In general by an easy recurrences on $k \geq 1$, we obtain:

$$
\begin{gathered}
a_{2 k}=(-1)^{k} a_{0}, \\
a_{2 k+1}= \begin{cases}-C a_{0}^{-1} a_{k}^{r} & \text { if } k \text { is even; } \\
-a_{k}^{r} / a_{0} C & \text { if } k \text { is odd. }\end{cases}
\end{gathered}
$$

We come now to compute the approximation exponent of $\alpha$. Set $u_{m}=\operatorname{deg} a_{m}$, $\lambda=\operatorname{deg} a_{0}$ and $\mu=\operatorname{deg} C$. So we have for $k \geq 1$

$$
u_{2 k}=\lambda
$$

and

$$
u_{2 k+1}= \begin{cases}(r-1) \lambda+\mu & \text { if } k \text { is even; } \\ r u_{k}-\lambda-\mu & \text { if } k \text { is odd. }\end{cases}
$$

Note that we will obtain this approximation exponent by computing $\limsup \left(\frac{u_{n}}{\sum_{0 \leq i \leq n-1} u_{i}}\right)$ 
and for this, we will follow [[9]. p 158] fairly closely.

We have for odd $k$,

$u_{2 k+1}=\lambda\left(r^{l}-r^{l-1}-\ldots-r-1\right)+\mu\left(r^{l-1}-r^{l-2}-\ldots-r-1\right)$ when $2^{l-1} \|(k+1)$.

For a given $n, n+1=2^{t}$ with $t>0$. We have that

$$
\begin{aligned}
& u_{n}=\lambda\left(r^{t}-r^{t-1}-\ldots-r-1\right)+\mu\left(r^{t-1}-r^{t-2}-\ldots-r-1\right)= \\
& \lambda\left(\frac{(r-2) r^{t}+1}{r-1}\right)+\mu\left(\frac{(r-2) r^{t-1}+1}{r-1}\right)
\end{aligned}
$$

and

$$
\begin{array}{r}
u_{1}+u_{2}+\ldots+u_{n-1}=\lambda\left(r^{t-1}-r^{t-2}-\ldots-r\right)+\mu\left(r^{t-2}-r^{t-3}-\ldots-r\right)= \\
\lambda\left(\frac{r^{t}-r}{r-1}\right)+\mu\left(\frac{r^{t-1}-r}{r-1}\right) .
\end{array}
$$

Therefore as $n$ runs through the numbers $r^{t}-1(t=1,2, \ldots)$, then

$$
\limsup \left(\frac{u_{n}}{\sum_{0 \leq i \leq n-1} u_{i}}\right)=r-2
$$

and then $v(\alpha)=1+r-2=r-1$.

We conclude this work by giving a sufficient condition on $A$ and $C$ to obtain a solution $\alpha$ of (1) with $v(\alpha)>1$, without giving the exact value of $v(\alpha)$. In fact, in [[7]. p 403], it has been proved that if an hyperquadratic element satisfying an equation of the type (1.1) (with $A D-B C=\Delta$ ), has a partial quotient other than the first with degree $>\frac{\operatorname{deg} \Delta}{r-1}$, then it will have unbounded partial quotients. According to the equation (1), we have $\operatorname{deg} \Delta=\operatorname{deg} A$.

Theorem 2.3. Let $\alpha$ be the irrational solution of (1). Suppose that there exist $H$ and $D \in \mathbf{K}[X]$ such that $D A-H^{r} C=1$ and $|H|=\left|\frac{A}{C}\right|$. Suppose moreover that $\operatorname{deg}\left(\left[\frac{A}{C}\right]-H\right)<\frac{(r-2) \operatorname{deg} A}{r(r-1)}$. Then $\alpha$ admits unbounded partial quotients.

Proof. Let $\left[\frac{A}{C}\right]=S$ then $|H|=|S|=|\alpha|$. We have :

$\left|\alpha-\frac{H^{r}}{D}\right|=\left|\frac{A \alpha^{r}}{C \alpha^{r}+1}-\frac{H^{r}}{D}\right|=\frac{\left|\alpha^{r}-H^{r}\right|}{|D||C||\alpha|^{r}}\left|=\frac{1}{|D||C|}\right| 1-\left.\frac{\alpha}{H}\right|^{r}$.

Consider that $\alpha=S+u_{1} X^{-1}+u_{2} X^{-2}+\ldots$ and $S-H=T$.

$$
\frac{\alpha}{H}=\frac{S+u_{1} X^{-1}+\ldots}{H}=\frac{H+T+u_{1} X^{-1}+\ldots}{H} .
$$

So if $T \neq 0$ then $\left|1-\frac{\alpha}{H}\right|^{r}=\frac{|T|^{r}}{|H|^{r}}$. Consequently, since $|D A|=\left|H^{r} C\right|$ then

$$
\left|\alpha-\frac{H^{r}}{D}\right|=\frac{1}{|C||D|} \frac{|T|^{r}}{|H|^{r}}=\frac{1}{|D|^{2}} \frac{|T|^{r}}{|A|} .
$$


Since $\operatorname{deg} T<\frac{(r-2) \operatorname{deg} A}{r(r-1)}$ then there exists a partial quotient of $\alpha$ of degree $\operatorname{deg} A-r \operatorname{deg} T>\frac{\operatorname{deg} A}{r-1}$. So we conclude that $\alpha$ admits unbounded partial quotients.

Now if $T=0$. Suppose that $u_{1} \neq 0$ then $\left|1-\frac{\alpha}{H}\right|^{r}=\frac{1}{|X|^{r}|H|^{r}}$. So we obtain :

$$
\left|\alpha-\frac{H^{r}}{D}\right|=\frac{1}{|D|^{2}} \frac{1}{|X|^{r}|A|} \text {. }
$$

Hence, there exists a partial quotient of $\alpha$ of degree $\operatorname{deg} A+r>\frac{\operatorname{deg} A}{r-1}$.

So we conclude that $\alpha$ admits unbounded partial quotients.

Example 2.2. Let Let $\alpha$ be the irrational solution of the equation (1) with $\mathbf{K}=\mathbb{F}_{3}$, $r=3, A=X^{4}+2 X^{2}+1$ and $C=X^{3}$. Then $\alpha$ admits unbounded partial quotients.

For this example, there exists $H=\left[\frac{A}{C}\right]=X$ and $D=X^{2}+1$ such that $D A-H^{3} C=1$, so by the previous Theorem we conclude that $\alpha$ admits unbounded partial quotients.

\section{Acknowledgement}

I would like to thank Alain Lasjaunias for helpful discussions.

\section{References}

[1] A. Lasjaunias, Continued fractions for hyperquadratic power series over finite field, Finite Fields Appl, 14(2008), 329-350.

[2] A. Lasjaunias, A Survey of Diophantine Approximation in Fields of Power Series, Monatsh. Math, 130(2000), 211-229.

[3] K. Mahler, On a theorem of Liouville in fields of positive characteristic, Canadian J Math, 1(1949), 397-400.

[4] B. de Mathan, Approximation exponents for algebraic functions, Acta Arithmetica, 60(1992), 359-370.

[5] B. de Mathan, Irrationality Measures and Transcendance in Positive Characteristic, Journal of Number Theory, 54(1995), 93-112.

[6] M. Mendes France, Sur les fractions continues limitées, Acta Arith, 23(1973), 207-215.

[7] W. Mills, D. Robbins, Continued fractions for certain algebraic power series, Journal of Number Theory, 23(1986), 388-404.

[8] M. Mkaouar, Sur les fractions continues des séries formelles quadratiques sur $\mathbb{F}_{q}(X)$, Acta Arithmetica, 97(2001), 241-251. 
[9] W. Schmidt, On continued fractions and diophantine approximation in power series fields, Acta Arithmetica, 95 (2000), 139-166.

[10] D. Thakur, Function Field Arithmetic, World Scientific, 2004.

[11] J. F. Voloch, Diophantine approximation in positive characteristic , Periodica Mathematica Hungarica, 19(3)(1988), 217-225.

Département de mathématiques, Faculté des sciences,

Université de Sfax, BP 802,

3038 Sfax, Tunisie

E.mail : ayedikhalil@yahoo.fr 